\title{
Aduhelm: The Best Hope for Alzheimer's Disease Patients or the Worst Decision the FDA Has Ever Made?
}

\author{
Markku Kurkinen* and Lloyd Tran \\ Biomed Industries, Inc., San Jose, CA, USA \\ Accepted 21 September 2021 \\ Pre-press 10 October 2021
}

On June 7, 2021, the US Food and Drug Administration (FDA) approved Aduhelm (aducanumab), the first new drug in 18 years for the treatment of Alzheimer's disease [1, 2]. Here, we revisit the considerable media attention, fierce debate, and controversy surrounding the Aduhelm approval, then discuss what it means to the 6.2 million people living with Alzheimer's disease at home or long-term care facilities in the US today.

Aducanumab (branded as Aduhelm), manufactured by Biogen, is one of the many anti-A $\beta$ monoclonal antibodies that has been shown to clear amyloid from the brain [3]. The approval of Aduhelm was based on two clinical trials EMERGE and ENGAGE, sponsored by Biogen, in study participants with mild to moderate cognitive impairment symptoms volunteering for the trials, one showing aducanumab delayed cognitive decline, and one showing it did not. The FDA approval of Aduhelm has been "a long and arduous journey" [2]. It is peculiar, though, that these studies and the results have not yet been published in any peer-reviewed paper.

In both Phase III trials, aducanumab caused adverse events of amyloid-related imaging abnormalities (ARIA), with vasogenic cerebral edema (ARIA-E) and brain microhemorrhages (ARIA-H), in $41 \%$ of study participants receiving the highest

\footnotetext{
*Correspondence to: Markku Kurkinen, Biomed Industries, Inc., San Jose, CA 95131, USA. E-mail: MKurkinen@ biomedind. com.
}

dose of aducanumab (10 mg/kg) compared with $10 \%$ on placebo. While the prescribing indications for aducanumab mandate routine MRI screening for ARIA, this will have a significant impact on risk/benefit calculations and the overall cost of aducanumab therapy.

On November 6, 2020, 11 members of the advisory committee for the FDA voted 8 to 1 ( 2 undecided) that data from the positive study was not sufficient to show the drug works, and voted 10 to 0 ( 1 undecided) that the positive study should not be considered as the evidence for the drug's approval, because the negative study had shown no effect, and if anything, the drug had enhanced cognitive decline [4].

When the FDA granted Accelerated Approval of aducanumab, it was based on the effect on the biomarker, reduction of brain amyloid, and on the possible clinical benefits observed in the EMERGE trial. FDA concluded that "the reduction in plaques is reasonably likely to result in clinical benefits." [5].

In the spring 2021, several members of the advisory committee expressed their concern on aducanumab in writing. On March 30, G. Galeb Alexander, Scott Emerson, and Aaron Kesselheim published a commentary in JAMA on the contradictory results of the two identical trials on the efficacy of aducanumab in slowing cognitive decline [6]. On April 7, David Knopman, Michael Jones, and David Greicius published a paper in Alzheimer's \& Dementia demonstrating convincingly there was no evidence in the Biogen data to correlate brain amyloid lowering with 
slowing cognitive decline, and wrote: "Post-hoc analysis suggesting that there may be an efficacy signal are unreliable.", and called for a definitive third Phase III trial "optimally designed and adequately powered to prove efficacy" [7]. In contrast, on May 10, Jeff Cummings and colleagues wrote in Alzheimer's Disease \& Therapy: "aducanumab achieves the standard of meaningful efficacy with adequate safety in early AD" and "We support providing persons with AD ... a first-generation drug with aducanumab's riskburden/benefit profile" [8].

On June 10, 2021, Aaron Kesselheim (Harvard Medical School and Brigham and Women's Hospital) resigned from the FDA advisory committee he had been serving for six years, following the resignation of Joel Perlmutter (Washington University School of Medicine) and David Knopman (Mayo Clinic) [4].

On June 16, 2021, Michael A. Carome, Director of the Public Citizen Health Research Group, sent a letter to Xavier Becerra, Secretary of the US Department of Health and Human Services (HHS) saying "The FDA's decision to approve aducanumab for anyone with Alzheimer's disease, regardless of severity, showed a stunning disregard for science, eviscerated the agency's standards for approving new drugs, and ranks as one of the most irresponsible and egregious decisions in the history of the agency." [9].

Alzheimer's disease is not one disease, but has many stages as it progresses over time, stages that differ in clinical signs and symptoms, and most likely in treatment options. On July 8, 2021, Biogen requested the FDA to indicate Aduhelm only in the treatment of people with mild to moderate cognitive impairment, the study population in the aducanumab trials [10].

Less than 3 weeks after the FDA approval of Aduhelm, US lawmakers announced an investigation into the approval and the drug's price of $\$ 56,000$ per patient-year, amid doubts if the drug works [11]. A few weeks later, Rep. Katie Porter (D-CA) urged HHS to investigate FDA and Biogen, to conduct "a full review" of the agency's communications with Biogen executives, lobbyists, and other stakeholders [12]. The FDA Acting Commissioner Janet Woodcock called for a federal investigation into the FDA approval that sparked the ire of US Congress, physicians, and public health advocates [13].

On July 12, 2021, the US Centers for Medicare and Medicaid Services initiated a process to decide how to cover the cost of Aduhelm. The process, which may last until April 2022, involves public hearings from patient advocacy groups, family members and other caregivers, medical professionals, and drug industry experts [14]. In the meantime, two House committees have asked Biogen to hand over documentation on a number of issues around the Aduhelm approval, including the unusual contacts between Biogen and FDA, under the code name Project Onyx, which may have helped Biogen to get Aduhelm approved [15].

To assure the general public, FDA requested Biogen to conduct a Phase IV confirmatory trial, which may take up to 9 years to complete. The results will not be available until 2030 to confirm or refute aducanumab can slow cognitive decline [5].

Trivial as it may seem, the EMERGE and ENGAGE trials were motivated by the amyloid hypothesis, which proposes Alzheimer's disease begins in the brain with $\mathrm{A} \beta$ peptides accumulation, aggregation, and amyloid formation. For 30 years, the amyloid hypothesis has almost singularly guided biomedical research, drug discovery, and clinical development in Alzheimer's disease. The FDA approval of Aduhelm is not a proof of the validity of the amyloid hypothesis. In fact, the amyloid hypothesis is not a hypothesis at all, because, in spite of all the observations and facts against the hypothesis, it seems it cannot be proven wrong. The amyloid hypothesis is too good to be true [16].

Aduhelm faces the headwinds from the lawmakers, Office of Inspection General, Medicare, private insurance payers, hospitals, medical professionals, and Alzheimer's disease patient advocacy groups. There are limited options available for the FDA and Biogen to correct the regulatory pathway of Aduhelm. In order to settle the controversy surrounding the clinical efficacy of Aduhelm, we suggest Biogen to expedite a third Phase III trial with the high dose of aducanumab $(10 \mathrm{mg} / \mathrm{kg})$ in APOE4 negative study population, similar to that which previously, in a post hoc analysis of the EMERGE data, had shown clinical efficacy.

The future of Aduhelm, the hope for the Alzheimer's disease patients, will now be in the hands of the US Congress, physicians, professional caregivers, and other stakeholders. The use of the accelerated approval pathway has confirmed the vital role of biomarkers in clinical trials of dementia that will enable new treatments to move faster into the clinic. Despite the controversy about the clinical data of Aduhelm and the FDA regulatory process, we anticipate a positive outlook that will encourage generation of new hypotheses and development of novel drugs that will bring hope to millions of patients of Alzheimer's disease. Fiat lux. 


\section{ACKNOWLEDGMENTS}

We appreciate the three anonymous reviewers for their concise and pertinent comments, contribution, and interest in this paper.

Authors' disclosures available online (https:// www.j-alz.com/manuscript-disclosures/21-5105r2).

\section{REFERENCES}

[1] FDA News, FDA Grants Accelerated Approval for Alzheimer's Drug, June 7, 2021. https://www.fda.gov/ news-events/press-announcements/fda-grants-acceleratedap-proval-alzheimers-drug?utm_medium=email\&utm source $=$ govdelivery.

[2] Yang P, Sun F (2021) Aducanumab: The first targeted Alzheimer's therapy. Drug Discov Ther 15, 166-168.

[3] Agadjanyan MG, Petrovsky N, Ghochikyan A (2015) A fresh perspective from immunologists and vaccine researchers: Active vaccination strategies to prevent and reverse Alzheimer's disease. Alzheimers Dement 11, 12461259.

[4] "Harvard expert quits FDA panel as furor over Biogen drug grows" by Robert Langreth, The Boston Globe, June 10, 2021.

[5] Patrizia Cavazzoni, Director, FDA Center for Drug Evaluation and Research- FDA's Decision to Approve New Treatment for Alzheimer's Disease, FDA News, June 7, 2021. https://www.fda.gov/drugs/news-events-humandrugs/fdas-decision-approve-new-treatment-alzheimersdisease.

[6] Alexander GC, Emerson S, Kesselheim AS (2021) Evaluation of aducanumab for Alzheimer disease: Scientific evidence and regulatory review involving efficacy, safety, and futility. JAMA 325, 1717-1718.

[7] Knopman DS, Jones D, Greicius MD (2021) Failure to demonstrate efficacy of aducanumab: An analysis of the EMERGE and ENGAGE trials as reported by Biogen, December 2019. Alzheimers Dement 17, 696-701.
[8] Cummings J, Aisen P, Lemere C, Atri A, Sabbagh M, Salloway S (2021) Aducanumab produced a clinically meaningful benefit in association with amyloid lowering. Alzheimers Res Ther 13, 98.

[9] Public Citizen-Press Release, June 16, 2021, Letter: Acting FDA Commissioner Woodcock, Other Top Officials Must Resign Following Indefensible Aducanumab Approval. https://www.citizen.org/news/letter-acting-fdacommissioner-woodcock-other-top-officials-must-resignfollowing-indefensible-aducanumab-approval/.

[10] Biogen, Press Release on July 8, 2021, FDA Approves Updated ADUHELMTM Prescribing Information to Emphasize Population Studied in Clinical Trials. https:// investors.biogen.com/news-releases/news-release-details/ fda-approves-updated-aduhelmtm-prescribing-informa tion-emphasize.

[11] Reuters, June 25, 2021, U.S. lawmakers to investigate approval, pricing of Alzheimer's drug from Biogen. https:// www.reuters.com/business/healthcare-pharmaceuticals/uslawmakers-investigate-approval-pricing-alzheimers-drugbiogen-2021-06-25/.

[12] Noah Higgins-Dunn, Lawmaker blasts FDA over Biogen's 'undue influence' in Aduhelm review, calls for HHS probFierce Pharma- Jul 7, 2021. https://www.fiercepharma.com/ pharma/rep-katie-porter-blasts-fda-over-biogen-s-undueinfluence-over-its-aduhelm-decision-calls.

[13] Janet Woodcock announcement on her Twitter account on July 9, 2021. https://twitter.com/DrWoodcockFDA/ status/1413540801934774283.

[14] Ricardo Alonso-Zaldivar and Matthew Perron, Associated Press, July 12, 2021, Medicare evaluating coverage for \$56,000 Alzheimer's drug. https://apnews.com/article/busi ness-health-government-and-politics-medicared68476e7c6f8eae33ab8a969feeeb1d1.

[15] Nathaniel Weixel, The Hill, July 9, 2021, FDA asks for federal investigation of Alzheimer's drug approval. https:// thehill.com/policy/healthcare/562289-fda-asks-forfederal-investigation-of-alzheimers-drug-approval.

[16] Kurkinen M (2017) The amyloid hypothesis is too good to be true. Alzheimers Dement Cogn Neurol 1, 1-9. 